Journal of Systems Science and Information

Jun., 2016, Vol. 4, No. 3, pp. 258-268

DOI: $10.21078 /$ JSSI-2016-258-11

\title{
Group Consensus of Second-Order Dynamic Multi-Agent Systems with Time-Varying Communication Delays and Directed Networks
}

\author{
Shuanghe MENG \\ School of Automation, Nanjing University of Science and Technology, Nanjing 210094, China \\ E-mail: h7spice@sina.cn
}

Lü XU

School of Automation, Nanjing University of Science and Technology, Nanjing 210094, China

E-mail: zdmisslv@163.com

Liang CHEN

School of Automation, Nanjing University of Science and Technology, Nanjing 210094, China

E-mail: 15651722230@163.com

\begin{abstract}
This paper studies the group consensus problem for second-order multi-agent dynamic systems with time-varying delays, where the agents in a network may reach one more consistent values asymptotically. The fixed network topology is in case of being directed and weakly connected. Based on algebraic graph theory and Lyapunov function approach, we propose some sufficient conditions for reaching group consensus. All the results are presented in the form of linear matrix inequalities(LMIs). A simulation example is provided to demonstrate the effectiveness of the theoretical analysis.
\end{abstract}

Keywords group consensus; second-order multi-agent systems; directed network; time-varying communication delays; LMIs

\section{Introduction}

In recent years, we have witnessed the rapid development and growing interest in cooperative control of multi-agent systems. Due to its extensive applications in unmanned aerial vehicle formation, robot formation control rendezvous in space, and other areas, many research have been focused on this project. In these applications, the states of all the agents are demand of reaching an agreement on some consistent value, which is called the consensus issue.

Consensus problem has been one of the vital issues in coordination control of multi-agent systems. By the pilot research work of Reynolds ${ }^{[1]}$ and Viseck ${ }^{[2]}$, periodical progress of the multi-agent system distributed coordination control has been made. Most of the researchers have studied consensus problems based on the point that how to design a suitable consensus algorithm or protocol. Olfati-Saber and Murray firstly introduced the theoretical framework for networked dynamic systems about the problems of solving consensus problems ${ }^{[3]}$. Many problems are related to consensus problem for multi-agent systems, for instance, collective behavior of flocks and swarms, formation control of multi-robots, and synchronization of coupled

Received January 22, 2016, accepted March 4, 2016

Supported by National Natural Science Foundation of China (612731200) 
oscillators $^{[4]}$. In [5-7], the consensus problems of multi-agent systems with first-order dynamics have been investigated from manifold perspectives. Due to the practical applications where multi-agent systems are with second-order dynamics, the more complicated and challenging consensus problem has received a great deal of attention, where agents are governed by both position and velocity states. The dynamical consensus algorithm for the second-order multiagent systems was proposed ${ }^{[8]}$, where all agents achieved the same consensus value. Based on discrete-time multi-agent system, where each agent stopped in the same positions with zero velocity, the static consensus algorithm under directed switching topologies with nonuniform time delays was given ${ }^{[9]}$. Ren showed that the existence of a directed spanning tree is a necessary rather than a sufficient condition to reach second-order consensus by giving a set of algorithms $^{[10]}$. In [11], Yu found that both the real and imaginary parts of the eigenvalues of the Laplacian matrix play key roles in reaching second-order consensus. Furthermore, a disagreement vector was introduced to analyze double-integrator dynamical multi-agent systems ${ }^{[12]}$. [13] studied the leader-following consensus problem of second-order multi-agent systems with switching and fixed topoplogies with non-uniform time-varying delays. High-order consensus of multi-agent systems attracted many researchers attention as well; see, for example, [14-19] and references there in.

Recently, a new concept of multi-agent group consensus problem, which differs from the classical consensus problem, has been pointed out and attracted lots and lots of attention within the control academical community ${ }^{[20-26]}$. In group consensus category, all agents in a multi-agent system are divided into multiple subgroups while each group will reach their own consensus values, which could be various for the changes of cooperative tasks and environment. At the side of traditional consensus problem, group consensus appears more complicated, which need some restriction and assumption. Yu and Wang firstly introduced group consensus to describe this kind of consensus ${ }^{[20]}$. The primary target of this issue is to desigh suitable consensus protocols or algorithms to make sure the states of the agents in each subgroup can reach the same value. In [20], Yu and Wang considered the situation with undirected topology under some assumptions, where algebratic condictions were derived and influence from any other group to the node is equal to zero. Later, the authors extended their research achievement to some other situations ${ }^{[21,22]}$. [21] discussed the average group consensus problem for multi-agent systems with directed topology. [22] considered the switching topologies and time delays for group consensus multi-agent systems, where the agents are described by single-intergrator dynamics, by promoting double-tree-form transformation. Leader-following approach and pinning control methods were investigated in [23] as well as group consensus problem was solved within nonlinear dynamics. Xie and Liang ${ }^{[24]}$ studied second-order group consensus with time delay by Lyapunov first method and Hopf bifurcation theory.

Motivated by the above discussions, we consider group consensus in second-order dynamic multi-agent system with communication time-varying delays. Firstly we extend and complement the group consensus results in [20] from single-order dynamic system to second-order dynamic system and obtain a consensus protocol based on group structure. Furthermore, the proposed protocol is extended from protocol with fixed time delay to protocol with communication time-varying delay. The Lyapunov function approach is used to analyze the group 
consensus problem for second-order multi-agent dynamical systems with communication timevarying delay, then a delay-dependent sufficient condition in form of LMI is given for reaching consensus such that we can judge the processed multi-agent system whether can reach a consensus by a feasibility testing of LMIs. The move has a significant significance for applying the theory to the practical applications due to the use of LMI technology.

The rest of this paper is organized as follows. In Section 2, we give some preliminaries and crucial formulation of group consensus system models. The main results of this paper are proposed in Section 3. Numerical simulations and conclusion are given in Sections 4 and 5, respectively.

\section{Problem Formulation and Preliminaries}

In this section, some basic concepts, results about graph theory are introduced and some problem formulations, definitions, assumptions and supporting lemmas are given to obtain the main results of the paper.

\subsection{Graph Theory}

The topology of a group multi-agent system is usually described by a graph. Let a weighted directed $G(V, E, A)$ model the interaction communication between $n+m$ agents, where $V=$ $\left\{v_{1}, v_{2}, \cdots, v_{n+m}\right\}$ is the set of nodes, $\varepsilon \subseteq V \times V$ is the set of edges, and $A=\left[a_{i j}\right] \in$ $R^{(n+m) \times(n+m)}$ is a weighted adjacency matrix with real adjacency elements $a_{i j}$. The node indexes belong to a finite index set $\Gamma=\{1,2, \cdots, n+m\}$. An edge of $G$ is denoted by $e_{i j}=\left(v_{i}, v_{j}\right)$. The adjacency elements associated with the edges of the graph are nonzero, i.e., $e_{i j} \in \varepsilon(G) \Leftrightarrow a_{i j} \neq 0$. Moreover, we assume $a_{i i}=0$ for all $i \in \Gamma$. The set of neighbors of the node $v_{i}$ is denoted by $N_{i}=\left\{v_{j} \in V:\left(v_{i}, v_{j}\right) \in \varepsilon(G)\right\}$. The Laplacian matrix of topology $G$ is defined by

$$
l_{i j}= \begin{cases}-a_{i j}, & j \neq i, \\ \sum_{k=1, k \neq i}^{n+m} a_{i k}, & j=i .\end{cases}
$$

\subsection{Problem Formulation}

Suppose that the multi-agent system consists of $n+m$ agents and each agent has the dynamics as follows:

$$
\left\{\begin{array}{c}
\dot{x}_{i}(t)=v_{i}(t), \\
\dot{v}_{i}(t)=u_{i}(t),
\end{array}\right.
$$

where $x_{i} \in R, v_{i} \in R$ denote the position and velocity of agent $i$, respectively, $u_{i} \in R$ is control input.

Without loss of generality, we consider the case of two groups, which can be expand to multiagent systems with more groups easily. Define $\Gamma_{1}=\{1,2, \cdots, n\}, \Gamma_{2}=\{n+1, n+2, \cdots, n+m\}$, $V_{1}=\left\{v_{1}, v_{2}, \cdots, v_{n}\right\}$ and $V_{2}=\left\{v_{n+1}, v_{n+2}, \cdots, v_{n+m}\right\}$ as the index sets and node sets to describe the two groups. Then, $\Gamma=\Gamma_{1} \cup \Gamma_{2}$ and $V=V_{1} \cup V_{2}$, respectively. Furthermore, let $N_{1 i}=\left\{v_{j} \in V_{1}:\left(v_{i}, v_{j}\right) \in \varepsilon(G)\right\}, N_{2 i}=\left\{v_{j} \in V_{2}:\left(v_{i}, v_{j}\right) \in \varepsilon(G)\right\}$ and $N_{i}=N_{1 i} \cup N_{2 i}$ be the neighbor sets of node $v_{i}$ in $V_{1}, V_{2}$, and $V$, respectively. Considering the communication 
time delay of agents would never be fixed, we improve the protocol ${ }^{[24]}$ and adopt the following control input:

$$
u_{i}(t)=\left\{\begin{array}{l}
-2 k v_{i}(t)+k \sum_{j \in N_{1 i}} a_{i j}\left[x_{j}\left(t-\tau_{i j}(t)\right)-x_{i}\left(t-\tau_{i j}(t)\right)\right] \\
+k \sum_{j \in N_{2 i}} a_{i j} x_{j}\left(t-\tau_{i j}(t)\right), \quad i \in \Gamma_{1}, \\
-2 k v_{i}(t)+k \sum_{j \in N_{2 i}} a_{i j}\left[x_{j}\left(t-\tau_{i j}(t)\right)-x_{i}\left(t-\tau_{i j}(t)\right)\right] \\
+k \sum_{j \in N_{1 i}} a_{i j} x_{j}\left(t-\tau_{i j}(t)\right), \quad i \in \Gamma_{2},
\end{array}\right.
$$

where $k>0, \tau_{i j}(t)>0$ means time-varying communication delay between agents. $a_{i j} \geq$ $0, \forall i, j \in \Gamma_{1}, a_{i j} \geq 0, \forall i, j \in \Gamma_{2}, a_{i j} \in R, \forall(i, j) \in I=\left\{(i, j): i \in \Gamma_{1}, j \in \Gamma_{2}\right\} \cup\{(i, j): j \in$ $\left.\Gamma_{1}, i \in \Gamma_{2}\right\}$. If $a_{i j}<0$, we consider that agent $j$ has a negative effect on agent $i$.

Definition 1 (Group consensus ${ }^{[20]}$ ) The multi-agent system (1) under protocol (2) is said to achieve second-order group consensus, if

$$
\begin{array}{lll}
\text { (i) } & \lim _{t \rightarrow+\infty}\left|x_{i}(t)-x_{j}(t)\right|=0, & \forall i, j \in \Gamma_{1}, \\
\text { (ii) } \lim _{t \rightarrow+\infty}\left|x_{i}(t)-x_{j}(t)\right|=0, & \forall i, j \in \Gamma_{2}, \\
\text { (iii) } \lim _{t \rightarrow+\infty}\left|v_{i}(t)\right|=0, & \forall i \in \Gamma .
\end{array}
$$

Definition 2 (Group average-consensus ${ }^{[20]}$ ) The multi-agent system (1) under protocol (2) is said to achieve second-order group average-consensus, if

$$
\begin{array}{lll}
\text { (i) } \lim _{t \rightarrow+\infty}\left|x_{i}(t)-x_{j}(t)\right|=\frac{1}{n} \sum_{j=1}^{n} x_{j}(0), & & \forall i, j \in \Gamma_{1}, \\
\text { (ii) } \lim _{t \rightarrow+\infty}\left|x_{i}(t)-x_{j}(t)\right|=\frac{1}{m} \sum_{j=n+1}^{n+m} x_{j}(0), & \forall i, j \in \Gamma_{2}, \\
\text { (iii) } \lim _{t \rightarrow+\infty}\left|v_{i}(t)\right|=0, & \forall i \in \Gamma .
\end{array}
$$

Assumption 1 We make the following propositions which means a balance of effect between two sub-networks:

$$
\begin{cases}\sum_{j=n+1}^{n+m} a_{i j}=0, & \forall i \in \Gamma_{1}, \\ \sum_{j=1}^{n} a_{i j}=0, & \forall i \in \Gamma_{2} .\end{cases}
$$

Assumption $2 \quad L$ has exactly two simple zero eigenvalues and all the other eigenvalues have positive real parts.

Assumption 3 The time-varying delays satisfy $0 \leq \tau_{i j}(t) \leq \tau$ and $\dot{\tau}_{i j}(t) \leq d<1$ for some known constants $\tau$ and $d$.

Remark 1 Assumption 1 means the sum of adjacent weights from every node in one group to all nodes in another group is identical at each time while Assumption 2 make the protocol of 
the system realisable for group consensus of multi-agent dynamic systems. Furthermore, while the communication time delays among the sub-groups are often long and should not be ignored due to the long transporting line, Assumption 3 is reasonable from the practical application attitude.

\section{Main Results}

In this section we use state transformation method and establish theorem to solve the group consensus problem of second-order systems with time-varying delays in directed and fixed topology.

In order to achieve the objective, we defined some error vector. Denote $a_{i}(t)=\frac{v_{i}(t)}{k}+$ $x_{i}(t), b_{1}(t)=\left[x_{2}(t)-x_{1}(t), a_{2}(t)-a_{1}(t), \cdots, x_{n}(t)-x_{1}(t), a_{n}(t)-a_{1}(t)\right]^{\mathrm{T}}, b_{2}(t)=\left[x_{n+2}(t)-\right.$ $\left.x_{n+1}(t), a_{n+2}(t)-a_{n+1}(t), \cdots, x_{n+m}(t)-x_{n+1}(t), a_{n+m}(t)-a_{n+1}(t)\right]^{\mathrm{T}}$ and $\delta(t)=\left[b_{1}^{\mathrm{T}}(t) b_{2}^{\mathrm{T}}(t)\right]^{\mathrm{T}}$. By using reduced-order Laplacian method proposed in [24], we can rewrite the system (1) under protocol control (2) as

$$
\dot{\delta}(t)=\left(I_{n+m-2} \otimes A\right) \delta(t)-\left(L_{k} \otimes B\right) \delta\left(t-\tau_{i j}(t)\right),
$$

where $A=\left[\begin{array}{cc}-k & k \\ k & -k\end{array}\right], B=\left[\begin{array}{ll}0 & 0 \\ 1 & 0\end{array}\right]$

$L_{k}=\left[\begin{array}{cccccc}l_{22}-l_{12} & \cdots & l_{2 n}-l_{1 n} & l_{2, n+2}-l_{1, n+2} & \cdots & l_{2, n+m}-l_{1, n+m} \\ \vdots & \ddots & \vdots & \vdots & \ddots & \vdots \\ l_{n 2}-l_{12} & \cdots & l_{n n}-l_{1 n} & l_{n+2,2}-l_{1, n+2} & \cdots & l_{n, n+m}-l_{1, n+m} \\ l_{n+2,2}-l_{n+1,2} & \cdots & l_{n+2, n}-l_{n+1, n} & l_{n+2, n+2}-l_{n+1, n+2} & \cdots & l_{n+2, n+m}-l_{n+1, n+m} \\ \vdots & \ddots & \vdots & \vdots & \ddots & \vdots \\ l_{n+m, 2}-l_{n+1,2} & \cdots & l_{n+m, n}-l_{n+1, n} & l_{n+m, n+2}-l_{n+1, n+2} & \cdots & l_{n+m, n+m}-l_{n+1, n+m}\end{array}\right]$.

Lemma 1 (Liang, et al. ${ }^{[2]}$ ) $L_{K}$ has the same eigenvalues as $L$ except two zero eigenvalues.

Lemma 2 (Boyd, et al. $\left.{ }^{[25]}\right)$ For a given matrix $S=\left[\begin{array}{ll}S_{11} & S_{12} \\ S_{12}^{\mathrm{T}} & S_{22}\end{array}\right]$, where $S_{11}$ and $S_{22}$ are square matrices, the following conditions are equivalent:

1) $S<0$;

2) $S_{11}<0, S_{22}-S_{12}^{\mathrm{T}} S_{11}^{-1} S_{12}<0$;

3) $S_{22}<0, S_{11}-S_{12} S_{22}^{-1} S_{12}^{\mathrm{T}}<0$.

Lemma 3 (Kwon, et al. ${ }^{[26]}$ ) For any given positive-definite matrix $M \in R^{n \times n}$ and a vector function $\delta:[\beta, \alpha] \rightarrow R^{n}$, the following inequalities hold:

$$
\begin{aligned}
& (\alpha-\beta) \int_{\beta}^{\alpha} \delta^{\mathrm{T}}(s) M \delta(s) \mathrm{d} s \geq\left(\int_{\beta}^{\alpha} \delta(s) \mathrm{d} s\right)^{\mathrm{T}} M\left(\int_{\beta}^{\alpha} \delta(s) \mathrm{d} s\right) \\
& \frac{(\alpha-\beta)^{2}}{2} \int_{\beta}^{\alpha} \int_{s}^{\alpha} \delta^{\mathrm{T}}(u) M \delta(u) \mathrm{d} u \mathrm{~d} s \geq\left(\int_{\beta}^{\alpha} \int_{s}^{a} \delta(u) \mathrm{d} u \mathrm{~d} s\right)^{\mathrm{T}} M\left(\int_{\beta}^{\alpha} \int_{s}^{a} \delta(u) \mathrm{d} u \mathrm{~d} s\right) .
\end{aligned}
$$

Theorem 1 Consider a second-order multi-agent system (1) and group consensus control protocol (2) satisfying Assumptions 1, 2 and 3, all the nodes of each group with comminication 
time-varying delay reach group consensus, if there exist matrices $T_{1}$ and $T_{2}$ with proper dimensions, positive definite and symmetric matrices $P, W, M, R, U$ and $\Theta=\left[\begin{array}{cc}\Theta_{11} & \Theta_{12} \\ * & \Theta_{22}\end{array}\right]>0$, such that the following LMIs hold:

$$
\begin{aligned}
& \Pi_{p}=\Pi_{p}^{\mathrm{T}}=\left[\Pi_{i j}\right]_{6 \times 6}<0, \\
& {\left[\begin{array}{ccc}
\Theta_{11} & \Theta_{12} & T_{1} \\
* & \Theta_{22} & T_{2} \\
* & * & \tau K_{1}^{\mathrm{T}} K_{2}
\end{array}\right]>0,}
\end{aligned}
$$

where

$$
\begin{aligned}
& \Pi_{11}=K_{1}^{\mathrm{T}} P+P K_{1}+K_{2}^{\mathrm{T}} P K_{2}+K_{1}^{\mathrm{T}} U K_{1}+T_{1}+T_{1}^{\mathrm{T}}-(v-u)^{2} R+\tau \Theta_{11} \\
& \Pi_{22}=-K_{2}^{\mathrm{T}} P K_{2}+K_{2}^{\mathrm{T}} U K_{2}-T_{2}-T_{2}^{\mathrm{T}}+\tau \Theta_{22} \\
& \Pi_{33}=-(v-u)^{2} W-U \\
& \Pi_{44}=-\frac{(v-u)^{2}}{4} W \\
& \Pi_{55}=-M \\
& \Pi_{66}=-R-W \\
& \Pi_{13}=(v-u) W \\
& \Pi_{12}=P K_{2}+K_{1}^{\mathrm{T}} U K_{2}-T_{1}+T_{2}+\tau \Theta_{22} \\
& K_{1}=I_{n+m-2} \otimes A \\
& K_{2}=L_{k} \otimes B
\end{aligned}
$$

and the remaining terms of $\Pi_{i j}$ are zeros.

Proof Choose a Lyapunov-Krasovskii functional candidate as follows:

$$
V_{i}(t)=\sum_{i=1}^{6} V_{i}(t)
$$

where

$$
\begin{aligned}
& V_{1}(t)=\delta^{\mathrm{T}}(t) P \delta(t), \\
& V_{2}(t)=\int_{t-\tau_{i j}(t)}^{t} \delta^{\mathrm{T}}(s) K_{2}^{\mathrm{T}} P K_{2} \delta(s) \mathrm{d} s \\
& V_{3}(t)=\int_{t-u}^{t} \dot{\delta}^{\mathrm{T}}(s) U \dot{\delta}(s) \mathrm{d} s \\
& V_{4}(t)=u \int_{-u}^{0} \int_{t+u}^{t} \delta^{\mathrm{T}}(s) M \delta(s) \mathrm{d} s \mathrm{~d} u, \\
& V_{5}(t)=(v-u) \int_{-v}^{-u} \int_{t+u}^{t} \delta^{\mathrm{T}}(s) R \delta(s) \mathrm{d} s \mathrm{~d} u, \\
& V_{6}(t)=\frac{(v-u)^{2}}{2} \int_{t-v}^{t-u} \int_{u}^{t-u} \int_{v}^{t-u} \dot{\delta}^{\mathrm{T}}(s) W \dot{\delta}(s) \mathrm{d} s \mathrm{~d} v \mathrm{~d} u,
\end{aligned}
$$


where $v, u$ are constants.

Calculating the time derivative of $V(t)$, we obtain

$$
\begin{aligned}
& \dot{V}_{1}(t)+\dot{V}_{2}(t)=\dot{\delta}^{\mathrm{T}}(t) P \delta(t)+\delta^{\mathrm{T}}(t) P \dot{\delta}(t)+\delta^{\mathrm{T}}(t) K_{2}^{\mathrm{T}} P K_{2} \delta(t) \\
& +\delta^{\mathrm{T}}\left(t-\tau_{i j}(t)\right) K_{2}^{\mathrm{T}} P K_{2} \delta\left(t-\tau_{i j}(t)\right) \\
& =\delta^{\mathrm{T}}(t)\left[K_{1}^{\mathrm{T}} P+P K_{1}+K_{2}^{\mathrm{T}} P K_{2}\right] \delta(t)-\delta^{\mathrm{T}}\left(t-\tau_{i j}(t)\right) K_{2}^{\mathrm{T}} P K_{2} \delta\left(t-\tau_{i j}(t)\right) \\
& +\delta^{\mathrm{T}}\left(t-\tau_{i j}(t)\right) K_{2}^{\mathrm{T}} P \delta(t)+\delta^{\mathrm{T}}(t) P K_{2} \delta\left(t-\tau_{i j}(t)\right), \\
& \dot{V}_{3}(t)=-\dot{\delta}^{\mathrm{T}}(t-u) U \dot{\delta}(t-u)+\dot{\delta}^{\mathrm{T}}(t) U \dot{\delta}(t) \\
& =-\dot{\delta}^{\mathrm{T}}(t-u) U \dot{\delta}(t-u)+\delta^{\mathrm{T}}(t) K_{1}^{\mathrm{T}} U K_{1} \delta(t)+\delta^{\mathrm{T}}(t) K_{1}^{\mathrm{T}} U K_{2} \delta\left(t-\tau_{i j}(t)\right) \\
& +\delta^{\mathrm{T}}\left(t-\tau_{i j}(t)\right) K_{2}^{\mathrm{T}} U K_{1} \delta(t)+\delta^{\mathrm{T}}\left(t-\tau_{i j}(t)\right) K_{2}^{\mathrm{T}} U K_{2} \delta\left(t-\tau_{i j}(t)\right), \\
& \dot{V}_{4}(t)=-u \int_{t-u}^{t} \delta^{\mathrm{T}}(s) M \delta(s) \mathrm{d} s \leq-\left[\int_{t-u}^{t} \delta(s) \mathrm{d} s\right]^{\mathrm{T}} M\left[\int_{t-u}^{t} \delta(s) \mathrm{d} s\right] \text {, } \\
& \dot{V}_{5}(t)=(v-u)^{2} \delta^{\mathrm{T}}(s) R \delta(s)-(v-u) \int_{t-v}^{t-u} \delta^{\mathrm{T}}(s) R \delta(s) \mathrm{d} s \\
& \leq(v-u)^{2} \delta^{\mathrm{T}}(s) R \delta(s)-\left[\int_{t-v}^{t-u} \delta(s) \mathrm{d} s\right]^{\mathrm{T}} R\left[\int_{t-v}^{t-u} \delta(s) \mathrm{d} s\right] \\
& \dot{V}_{6}(t)=\frac{(v-u)^{2}}{4} \dot{\delta}^{\mathrm{T}}(t-u) W \dot{\delta}(t-u)-\frac{(v-u)^{2}}{2} \int_{t-v}^{t-u} \int_{u}^{t-u} \dot{\delta}^{\mathrm{T}}(s) W \dot{\delta}(s) \mathrm{d} s \mathrm{~d} u \\
& \leq \frac{(v-u)^{2}}{4} \dot{\delta}^{\mathrm{T}}(t-u) W \dot{\delta}(t-u) \\
& -\left[(v-u) \delta(t-u)-\int_{t-v}^{t-u} \delta(u) \mathrm{d} u\right]^{\mathrm{T}} W\left[(v-u) \delta(t-u)-\int_{t-v}^{t-u} \delta(u) \mathrm{d} u\right] .
\end{aligned}
$$

By the Leibniz-Newton formula, we have

$$
\int_{t-\tau_{i j}(t)}^{t} \dot{\delta}(\beta) \mathrm{d} \beta=\delta(t)-\delta\left(t-\tau_{i j}(t)\right)
$$

Then, for any $T_{1}$ and $T_{2}$ with appropriate dimensions, we have

$$
2\left[\delta^{\mathrm{T}}(t) T_{1}+\delta^{\mathrm{T}}\left(t-\tau_{i j}(t)\right) T_{2}\right]\left[\delta(t)-\int_{t-\tau_{i j}(t)}^{t} \dot{\delta}(\beta) \mathrm{d} \beta-\delta^{\mathrm{T}}\left(t-\tau_{i j}(t)\right)\right]=0
$$

Obviously, for any matrix $\Theta \geq 0$, it holds that

$$
\tau\left[\begin{array}{c}
\delta(t) \\
\delta\left(t-\tau_{i j}(t)\right)
\end{array}\right]^{\mathrm{T}} \Theta\left[\begin{array}{c}
\delta(t) \\
\delta\left(t-\tau_{i j}(t)\right)
\end{array}\right] \geq \int_{t-\tau_{i j}(t)}^{t}\left[\begin{array}{c}
\delta(t) \\
\delta\left(t-\tau_{i j}(t)\right)
\end{array}\right]^{\mathrm{T}} \Theta\left[\begin{array}{c}
\delta(t) \\
\delta\left(t-\tau_{i j}(t)\right)
\end{array}\right] \mathrm{d} \beta
$$


Combining Equations (10)-(17) yields

$$
\begin{aligned}
& \dot{V}_{i}(t) \leq\left[\begin{array}{c}
\delta(t) \\
\delta\left(t-\tau_{i j}(t)\right) \\
\delta(t-u) \\
\dot{\delta}(t-u) \\
\int_{t-u}^{t} \delta^{\mathrm{T}}(s) \mathrm{d} s \\
\int_{t-v}^{t-u} \delta^{\mathrm{T}}(s) \mathrm{d} s
\end{array}\right]^{\mathrm{T}}\left[\begin{array}{cccccc}
\Pi_{11} & \Pi_{12} & \Pi_{13} & \Pi_{14} & \Pi_{15} & \Pi_{16} \\
* & \Pi_{22} & \Pi_{23} & \Pi_{24} & \Pi_{25} & \Pi_{26} \\
* & * & \Pi_{33} & \Pi_{34} & \Pi_{35} & \Pi_{36} \\
* & * & * & \Pi_{44} & \Pi_{45} & \Pi_{46} \\
* & * & * & * & \Pi_{55} & \Pi_{56} \\
* & * & * & * & * & \Pi_{66}
\end{array}\right]\left[\begin{array}{c}
\delta(t) \\
\delta\left(t-\tau_{i j}(t)\right) \\
\delta(t-u) \\
\dot{\delta}(t-u) \\
\int_{t-u}^{t} \delta^{\mathrm{T}}(s) \mathrm{d} s \\
\int_{t-v}^{t-u} \delta^{\mathrm{T}}(s) \mathrm{d} s
\end{array}\right] \\
& -\int_{t-\tau_{i j}(t)}^{t}\left[\begin{array}{c}
\delta(t) \\
\delta\left(t-\tau_{i j}(t)\right) \\
\dot{\delta}(\beta)
\end{array}\right]^{\mathrm{T}}\left[\begin{array}{ccc}
\Theta_{11} & \Theta_{12} & T_{1} \\
* & \Theta_{22} & T_{2} \\
* & * & \tau K_{1}^{\mathrm{T}} K_{2}
\end{array}\right]\left[\begin{array}{c}
\delta(t) \\
\delta\left(t-\tau_{i j}(t)\right) \\
\dot{\delta}(\beta)
\end{array}\right] \text {. }
\end{aligned}
$$

Then, by Lemma 2, Equations (7) and (8), we can get $\dot{V}(t)<0$. According to the definition of Lyapunov-Krasovskii functional candidate (9), system (6) is asymptotically stable, which implies that system (1) would achieve group consensus. This completes the proof.

Remark 2 For group consensus multi-agent systems with communication time-varying delay and directed topology, protocol (2) ensure couple-group consensus, which indicates the extension of the issue from single-integrator dynamics to double-integrator situations is not trival. Furthermore, it is indicated that both time delay bound and Laplacian matrix play key roles in reaching group consensus.

\section{Numerical Example}

In this section, to illustrate the effectiveness of the results proposed in previous section, we consider a directed graph with 6 agents indexed by 1 to 6 , respectively, in which Agents 1 to 4 are in group I and Agents 5 and Agents 6 belong in group II as shown in Figure 1. Hence, the Laplacian matrix $L$ of the system is given by

$$
L=\left[\begin{array}{cccccc}
2 & -2 & 0 & 0 & 0 & 0 \\
0 & 2 & -2 & 0 & -1 & 1 \\
-2 & 0 & 2 & 0 & 1 & -1 \\
-1 & 0 & 0 & 1 & 0 & 0 \\
0 & -1 & 1 & 1 & 3 & -4 \\
0 & 0 & 0 & 0 & -1 & 1
\end{array}\right]
$$




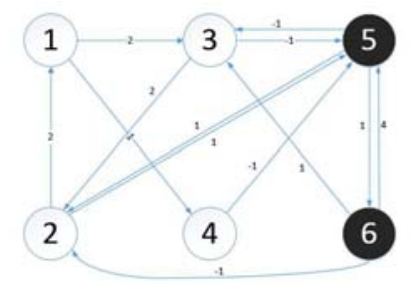

Figure 1 The directed topology with six agents

It is easy to obtain that $L$ has eigenvalues $\lambda_{1}=\lambda_{2}=0, \lambda_{3}=4.4557, \lambda_{4}=2.7+1.177 \mathrm{i}$, and $\lambda_{5}=2.7-1.177 \mathrm{i}$. $L$ has zero eigenvalues whose algebraic multiplicity is exactly two apparently, and the rest of the eigenvalues have positive real parts. We choose $k=1.2, \mu=0.1$, $v=0.35, d=0.25$ and define $\tau_{i j}(t)=\tau|\sin (t)|$ for convenience. Let the initial state and velocity be $x(0)=[1,100,-2,50,3,-1]^{\mathrm{T}}, v(0)=[1,2,1.5,3,2.2,0.6]^{\mathrm{T}}$. When $\tau=0.25$, Figure 2 shows the states trajectories of all six agents and Figure 3 shows the velocity trajectories of all agents for the multi-agent system.

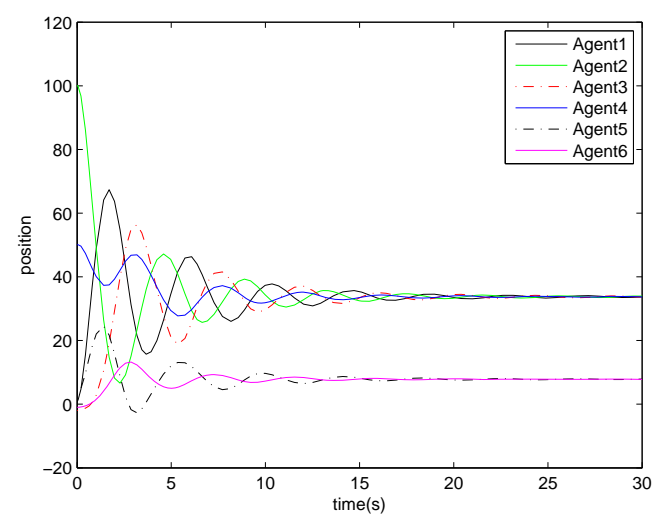

Figure 2 The trajectories of position $x_{i}$ when $\tau=0.25$

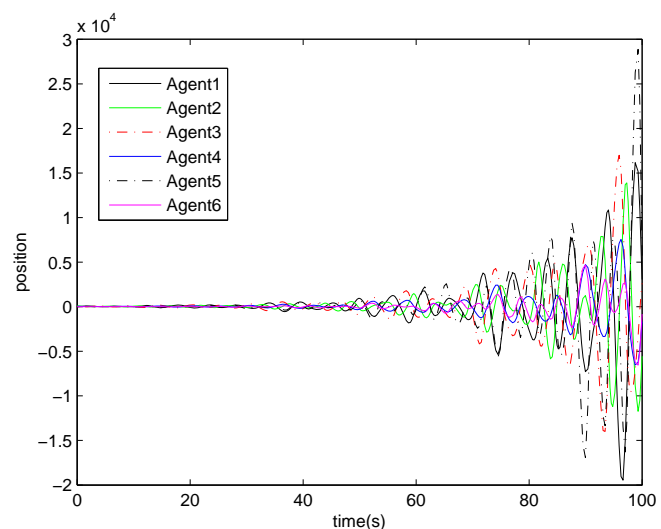

Figure 4 The trajectories of position $x_{i}$ when $\tau=0.87$

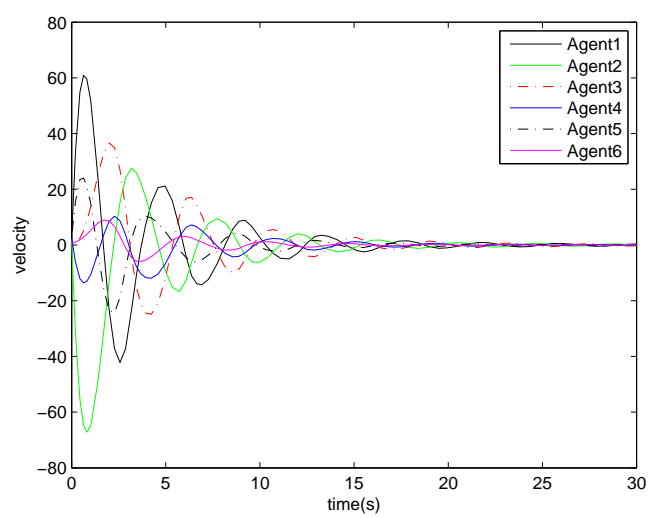

Figure 3 The trajectories of velocity $v_{i}$ when $\tau=0.25$

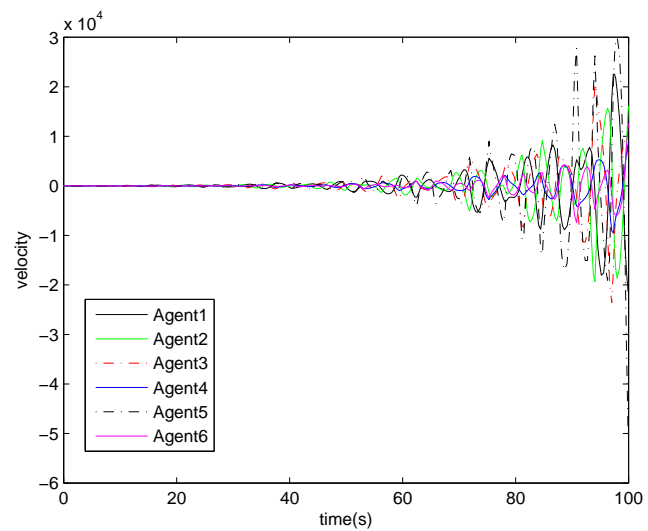

Figure 5 The trajectories of velocity $v_{i}$ when $\tau=0.87$ 
From Figure 2, it can be observed that states $x_{1}$ to $x_{4}$ converge to one consensus value, whereas the states $x_{5}$ and $x_{6}$ converge to another consensus value. Moreover, it can be seen from Figure 3 that the states $v_{1}$ to $v_{6}$ converge to one consensus value asymptotically. That is to say, the group consensus can be achieved. Furthermore, when $\tau \leq 0.25$, LMIs in Theorem 1 are feasible, which means that the system can achieve group consensus asymptotically. By changing parameters and increasing communication time delay bound, situations would change and when it comes to $\tau=0.52$, LMIs in Theorem 1 are unfeasible while the systems still can reach group consensus. It represents that our result still has the conservativeness to some extent. When $\tau=0.73$, it can be concluded from Figure 4 and Figure 5 that neither the position nor velocity can get consensus while LMIs are unfeasible which means the system appears divergency and cannot reach group consensus.

\section{Conclusions}

In this paper, the group consensus problem for second-order multi-agent dynamic systems with communication time-varying delays has been investigated, where the agents in a network may reach one more consistent values asymptotically. The delay-dependent sufficient conditions of achieving group consensus have been proposed based on Lyapunov function method and LMIs. Finally, simulation results are provided to verify the effectiveness of the proposed condition for agreement in networks with communication time-delays.

\section{References}

[1] Reynolds C. Flocks, herds, and schools: A distributed behavioral model. Computers \& Graphics, 1987, 21(4): $25-34$.

[2] Viseck T, Czirók A, Ben J E, et al. Novel type of phase transition in a system of self-driven particles. Physical Review Letters, 1995, 75(6): 1226-1229.

[3] Olfati-Saber R, Murray R M. Consensus protocols in networks of agents with switching topology and time-delays. IEEE Transactions on Automatic Control, 2004, 49(9): 1520-1533.

[4] Bullo F, Cortés J, Piccoli B. Special issue on control and optimization in cooperative networks. SIAM Journal on Control \& Optimization, 2009, 48(1): vii.

[5] Jadbabaie A, Lin J, Morse A S. Erratum: Coordination of groups of mobile autonomous agents using nearest neighbor rules. IEEE Transactions on Automatic Control, 2003, 48(9): 1675-1675.

[6] Sun Y G, Wang L. Consensus of multi-agent systems in directed networks with nonuniform time-varying delays. IEEE Transactions on Automatic Control, 2009, 54(7): 1607-1613.

[7] Tian Y P, Liu C L. Consensus of multi-agent systems with diverse input and communication delays. IEEE Transactions on Automatic Control, 2008, 53(9): 2122- 2128.

[8] Yu W, Zheng W X, Chen G, et al. Second-order consensus in multi-agent dynamical systems with sampled position data. Automatica, 2011, 47(7): 1496-1503.

[9] Lin P, Jia Y M. Consensus of second-order discrete-time multi-agent systems with nonuniform time-delays and dynamically changing topologies. Automatica, 2009, 45(9): 2154-2158.

[10] Ren W, Atkins E. Distributed multi-vehicle coordinated control via local information exchange. International Journal of Robust \& Nonlinear Control, 2007, 17(10-11): 1002-1033.

[11] Yu W, Chen G, Cao M. Some necessary and sufficient conditions for second-order consensus in multi-agent dynamical systems. Automatica, 2010, 46(6): 1089-1095.

[12] Xie G, Wang L. Consensus control for a class of networks of dynamic agents. International Journal of Robust \& Nonlinear Control, 2007, 17(10-11): 941-959.

[13] Zhu W, Cheng D. Cheng, Leader-following consensus of second-order agents with multiple time-varying delays. Automatica, 2010, 46(12): 1994-1999. 
[14] Ren W, Moore K, Chen Y Q. High-Order consensus algorithms in cooperative vehicle systems. Proceedings of the 2006 IEEE International Conference on ICNSC '06. 2006: 457-462.

[15] Xiao F, Wang L. Consensus problems for high-dimensional multi-agent systems. IET Control Theory \& Applications, 2007, 1(3): 830-837.

[16] Wang J, Liu Z, Hu X. Consensus of high order linear multi-agent systems using output error feedback. Proceedings of the 48th IEEE Conference on Decision and the 28th Chinese Control Conference, 2009: 3685-3690.

[17] Seo J H, Shim H, Back J. High-order consensus of MIMO linear dynamic systems via stable compensator. Proceedings of European Control Conference (ECC), 2009: 773-778.

[18] Zhai G, Okuno S, Imae J, et al. A new consensus algorithm for multi-Agent systems via dynamic output feedback control. Control Applications \& Intelligent Control, 2009: 890-895.

[19] Zhou B, Lin Z. Consensus of high-order multi-agent systems with input and communication delays-state feedback case. Proceedings of American Control Conference (ACC), 2013: 4027-4032.

[20] Yu J, Wang L. Group consensus of multi-agent systems with undirected communication graphs. Proceedings of Asian Control Conference (ACC), 2009: 105-110.

[21] $\mathrm{Yu}$ J, Wang L. Group consensus of multi-agent systems with directed information exchange. International Journal of Systems Science, 2012, 43(2): 334-348.

[22] Yu J, Wang L. Group consensus in multi-agent systems with switching topologies and communication delays. Systems \& Control Letters, 2010, 59(6): 340-348.

[23] Ma Q, Wang Z, Miao G. Second-order group consensus for multi-agent systems via pinning leader-following approach. Journal of the Franklin Institute, 2014, 351(3): 1288-1300.

[24] Xie D, Liang T. Second-order group consensus for multi-agent systems with time delays. Neurocomputing, 2015, 153: 133-139.

[25] Boyd B P, Ghaoui L E, Feron E, et al. Linear matrix inequalities in system and control theory. Society for Industrial and Applied, Philadelphia, 1994.

[26] Kwon O M, Lee S M, Ju H P, et al. New approaches on stability criteria for neural networks with interval time-varying delays. Applied Mathematics \& Computation, 2012, 218(19): 9953-9964. 